\title{
Le devenir socioéconomique des mères adolescentes, 10 ans plus tard
}

\section{The socioeconomic future of the teenage mothers, 10 years later}

\author{
A. Bouillaut, G. Grandazzi, N. Brielle
}

\begin{abstract}
Résumé Le devenir socioéconomique des mères adolescentes dix ans plus tard est un sujet peu traité. Nous avons donc mené des entretiens semi-dirigés auprès de neuf femmes ayant accouché en 2004 au CHU de Caen, alors qu'elles avaient entre 15 et 19 ans. Il ressort de cette étude que le devenir de ces femmes d'un point de vue professionnel et relationnel (conjugal et amical) dépend notamment de leur typologie de grossesse. Concernant le devenir obstétrical et le devenir de l'enfant, nous pouvons globalement dire que ces femmes ont eu en moyenne trois enfants et que leurs enfants ont eu un bon développement. Enfin, l'ensemble des femmes interrogées semble avoir conservé une bonne estime de soi, mais une partie avoue que cela a été une expérience difficile et toutes ne réitéreraient pas cette expérience et ne la souhaitent pas à leurs enfants.
\end{abstract}

\section{Mots clés}

Mères adolescentes ; Dix ans ; Devenir socioéconomique ; Parcours ; Évolution

Summary The socioeconomic future of the teenage mothers 10 years later is a relative unknown subject. So, we did semi-directed interviews to 9 women aged between 15 and 19 years old that gave birth in 2004 at the CHU in Caen. The study shows that the professional and relational (conjugal and friendship) future of these women mainly depend on their pregnancy typology. About others points, that is mean obstetrical future and child future, it also appears that the children generally have a good development and that the teenagers end up being mother of three children. Most of the respondents have a high sense of selfesteem, but some of them admit that it was not always easy and they would not repeat the experience or would not want this happening to their own children.

\section{Keywords}

Teen mothers; Ten years; Socioeconomic future; Professional; Wellbeing; Evolution

\section{Introduction}

En ce début du XXI ${ }^{e}$ siècle, médias et politiques s'emparent régulièrement de la question de la grossesse à l'adolescence. Elle est considérée comme un problème de santé publique, contre lequel les institutions essaient de lut- ter pour en diminuer l'incidence, et donc les conséquences qu'elle est supposée entraîner. Mais qu'en est-il réellement du devenir socioéconomique de ces mères adolescentes?

\section{Définition et concept de l'adolescence}

Notion récente historiquement, les bornes de l'adolescence sont difficiles à poser. Le Petit Larousse définit cette période comme : «Période de la vie entre l'enfance et l'âge adulte, pendant laquelle se produit la puberté et se forme la pensée abstraite " [1]. L'Organisation mondiale de la santé, elle, pose des bornes numériques à l'adolescence, considérant que cette période se déroule entre 10 et 19 ans et correspond à une «période de croissance et de développement humain » marquée par de nombreux changements [2].

L'adolescence est une période de conflit interne, où l'adolescent n'a pas un statut socialement défini. Il n'est plus un enfant, mais n'est pas encore un adulte. Cette absence d'identification à un statut social bien défini provoque des incertitudes se traduisant par des comportements puérils à certains moments, et matures à d'autres [3].

\section{Les raisons de la grossesse adolescente}

La sociologue C. Le Van a proposé une typologie des grossesses adolescentes.

\section{La grossesse rite d'initiation}

Elle est attendue, en tout cas non évitée. Les adolescentes pouvant être inclues dans cette catégorie ont un désir de grossesse et non pas un désir d'enfant, le but étant de tester leur partenaire face à leur rôle de futur père. Ce type de grossesse aboutit généralement à une IVG qui semble bien 
vécue par l'adolescence [4].

\section{Grossesse S.O.S}

Ces grossesses surviennent après des conduites contraceptives à risques, mais ne sont pas pour autant programmées par les adolescentes. Les grossesses apparaissent dans un contexte familial instable comme le divorce des parents, une situation de maltraitance, des difficultés relationnelles... Ces grossesses sont un moyen de solliciter l'attention de leurs parents. Elles se terminent le plus souvent par une IVG qui est mal vécue par l'adolescente [4].

\section{La grossesse insertion}

Dans ce cas, la grossesse est programmée et fait suite à une réflexion mûre et un arrêt de la contraception. Ces jeunes femmes et ces couples ont un réel désir d'enfant et de devenir parents. Les grossesses arrivent cependant dans un contexte d'échec scolaire ou de rupture affective avec leur famille. Le futur enfant devient alors le moyen de «pallier le vide de leur existence, d'acquérir un statut socialement reconnu et approuvé par l'entourage » [4]. Cette grossesse permet alors de mettre un terme à l'enfance et aux souffrances qui y sont associées [5].

\section{Grossesse identité}

Ici, la grossesse n'est pas planifiée de manière consciente. Lorsque la grossesse survient, elle est expliquée comme étant un accident, ce qui permet d'établir un compromis entre leur désir inconscient d'enfant et l' « interdit social qui pèse sur la maternité à leur âge ». Par ailleurs, le but de cette maternité est de refaire surface après avoir été dans une impasse scolaire et un désinvestissement généralisé Cette grossesse permet aux adolescentes d'éprouver un sentiment de revalorisation [4].

\section{Grossesse accidentelle}

La grossesse est non désirée, imprévue et fait suite à une erreur de contraception. La découverte de la grossesse est alors généralement tardive et l'adolescente peut prendre la décision ou non, de poursuivre cette grossesse. Cela dépend notamment de la stabilité du couple, de la situation financière et familiale ainsi que du désir de poursuite des études de la jeune femme [4].

\section{Grossesse culturelle}

Dans les cultures africaines, gitanes et maghrébines, le fait de porter un enfant jeune est valorisant et représente un rite de passage au statut d'adulte [6]. De plus, comme l'explique la psychologue Joëlle Berrewaerts, les femmes africaines mineures sont «psychologiquement déjà des femmes adultes culturellement et socialement » [7].

\section{La grossesse adolescente}

Des facteurs qui influencent les raisons d'une grossesse précoce sont variables. Malgré tout, on retrouve des facteurs favorisant l'accès à une maternité précoce. Comme le comportement contraceptif inadapté. Mais l'entourage joue un rôle important dans la survenue d'une grossesse adolescente. Pour Brent C. Miller, une famille avec un lien parent-enfant solide, la supervision des activités des enfants et la transmission des valeurs parentales concernant les relations sexuelles à l'adolescence permettent de diminuer le risque de grossesse précoce. A contrario, la monoparentalité, avoir des frères et sœurs sexuellement actifs et/ou des parents peu présents, ainsi qu'avoir un faible réseau ami- cal augmentent le risque d'avoir une grossesse adolescente [7]. Pour finir, le niveau socioéconomique faible semble le facteur le plus favorisant $[3,4,7,6,9]$

\section{Devenir des mères adolescentes}

Très peu d'études se sont intéressées au devenir des mères adolescentes ou alors, avec un recul très court, d'un an.

\section{Le devenir maternel}

Le risque de former une famille monoparentale concerne $70 \%$ des jeunes femmes ayant eu une grossesse adolescente $[10,11]$, avec un risque d'être mère isolée à 33 ans multi- plié par 8 [9]. Ce sont également des femmes moins souvent mariées [12].

Ensuite, socialement, Christine Loignon dans son étude pense que la grossesse adolescente provoque un isolement social [7]. Par ailleurs Bailey a retrouvé que $75 \%$ des jeunes femmes n'avaient pas perçu de détérioration de leur relation parent-enfant un an après leur grossesse [13].

En ce qui concerne le devenir social, une majorité des études montre une déscolarisation des adolescentes lors de leur grossesse $[5,7,9,10]$. En effet, l'arrêt de l'école et la nonobtention du baccalauréat concernent $67 \%$ des femmes entre 15 et 19 ans contre $34 \%$ des femmes de plus de 20 ans [7]. Seules $12,3 \%$ des jeunes filles poursuivent leur scolarité pendant leur grossesse, d'ailleurs cela multiplie par 6 leur chance de continuer les études après l'accouchement [13]. À l'inverse, certaines adolescentes avaient décroché du système scolaire avant d'être enceintes [7]. Selon Bailey, les adolescentes ayant eu une grossesse non désirée ont trois fois plus de chances de retourner à l'école après la naissance du bébé que les adolescentes ayant une grossesse désirée. De même, plus l'adolescente est âgée, plus elle risque de mettre un terme à sa scolarité [13].

Pour le devenir professionnel, le fait que les adolescentes soient moins diplômées les expose à une précarité professionnelle. La grossesse adolescente augmente le risque de chômage [11], d'emplois sous-payés et de pauvreté [9,12]. De plus, les mères ayant arrêté l'école trouvent un emploi seulement4à 6 ans plus tard [11].

Après la naissance de leur enfant, les études montrent que les jeunes mères ont une meilleure estime d'elles- mêmes $[7,11,13]$. Elles jugent cette grossesse comme ayant eu un impact positif sur leur vie [13].

Enfin, la grossesse suivant la première naissance arrive généralement deux ans après dans $35 \%$ des cas. Mais selon Bailey, sur l'ensemble des adolescentes enceintes (faussecouche, IVG, MFIU compris), $21 \%$ des adolescentes étaient de nouveau enceintes, avaient accouché d'un autre enfant ou avaient eu un avortement l'année suivante [13].

\section{Devenir de l'enfant}

Selon le psychanalyste Winnicott, les «mères adolescentes n'ont pas moins de dons que d'autres pour devenir des mères suffisamment bonnes ». Pour autant selon la littérature, le devenir de ces enfants n'est pas positif sur tous les points. En effet, 47,7 \% des enfants ne verraient a priori jamais leur père tandis que $35 \%$ auraient un père très présent [11]. Les 
liens mère-enfant, eux, « résistent plutôt mal à l'épreuve du temps » [12].

Par ailleurs, ces enfants auraient un retard du développement cognitif $[7,9,12]$, un moins bon niveau d'études, un risque plus important d'être impliqués dans des activités criminelles [7]. Pour finir, $25 \%$ d'entre eux sont confiés à une institution ou une famille d'accueil [11].

\section{Matériels et méthode}

\section{Objectifs}

Le but de ce travail était de rendre compte du devenir socioéconomique des jeunes mères à long terme et plus précisément, de savoir si devenir mère avant l'âge de 20 ans prédispose ces jeunes femmes à un avenir plus incertain.

\section{Matériels et méthode}

Afin de répondre à cette problématique, nous avons réalisé une étude qualitative avec des entretiens semidirigés.

Pour cela, nous nous sommes penchés sur les dossiers obstétricaux informatisés des jeunes femmes ayant accouché en 2004 au CHU de Caen. La population concernée était constituée de toutes les adolescentes âgées entre 10 et 19 ans ayant accouché ou consulté au $\mathrm{CHU}$ de Caen en 2004. Parmi cette population, nous avons cependant exclu : les patientes ne parlant pas le Français en 2004 ou actuelle- ment, les patientes ne résidant plus en Basse-Normandie, les patientes sous curatelle/tutelle, et les patientes dont l'issue de la grossesse a été une IMG, une MFIU ou un décès périnatal connu.

Nous avons donc étudié 138 dossiers obstétricaux. Sur l'ensemble des dossiers, 11 patientes ont été exclues (1 patiente sous tutelle, 4 patientes non francophones, 4 patientes ne vivant plus en Basse-Normandie, 1 patiente ayant eu une IMG, 1 patiente ayant eu une MFIU).

Les 127 femmes restantes ont été recontactées par télé- phone à partir des coordonnées les plus récentes retrouvées. Parmi cette population, 107 femmes ont été perdues de vue, 7 femmes ont refusé de témoigner, 4 femmes ne se sont pas présentées au rendez-vous, 9 femmes ont accepté de témoigner de leur parcours.

\section{Résultats}

Les femmes interrogées avaient un âge compris entre 15 et 19 ans au moment de leur grossesse précoce. Une mère avait 15 ans en 2004, 3 avaient 17 ans, 4 avaient 18 ans et une était âgée de 19 ans. L'ensemble des femmes était primigeste en 2004.

Nous avons réparti cette population en fonction de la typologie de leur grossesse : 3 femmes correspondaient au profil des grossesses « insertion », 2 au profil des grossesses

« identités » et 4 au profil des grossesses « accidentelles ». Aucune des femmes interrogées ne correspondait au profil des grossesses « culturelles ».

\section{Le devenir relationnel}

En ce qui concerne le devenir conjugal, nous avons remarqué que presque la moitié (4/9) des femmes est toujours avec le même compagnon depuis la naissance de leur premier enfant. Cependant, on peut distinguer 2 catégories de couples : les couples qui n'ont jamais vécu de séparation $(2 / 4)$ et qui sont soit mariées soit pacsées ; les couples qui se sont séparés $(2 / 4)$ pendant une courte période (pendant une grossesse) et qui vivent désormais en concubinage.

Pour les femmes n'étant plus en couple avec le même partenaire (5/9), la séparation a eu lieu pour la plupart rapidement après la naissance de l'enfant et suite à un désir de la femme d'y mettre un terme. De même, les relations avec l'ex-conjoint sont dans la majorité des cas difficiles voire inexistantes (4/5). Pour autant, la majorité d'entre elles ne sont pas mères-célibataires puisque qu'elles vivent en couple depuis au moins 4 ans.

Ensuite, concernant les relations familiales, dans la majorité des cas la famille a eu du mal à accepter la grossesse, mais sur une durée très courte de quelques jours $(8 / 9)$. Actuellement, pour les femmes concernées, la relation familiale est bonne.

Pour finir, concernant le cercle amical, deux populations se distinguent. Dans la première population, les jeunes filles ont conservé le même cercle amical depuis 10 ans (4/9) et dans la seconde, les jeunes femmes ont un nouveau cercle amical (4/9) qu'elles justifient par le fait que la maternité a provoqué un changement de centres d'intérêt chez elle.

\section{Devenir scolaire et professionnel}

À propos de la scolarité, nous retrouvons là aussi deux types d'évolutions. Tout d'abord pour les grossesses non planifiées, on remarque que toutes les jeunes femmes ont, soit continué leurs études pendant la grossesse, soit ont repris les cours après (4/9). Dans tous les cas, elles sont en possession d'un diplôme (BEP ou CAP dans la majorité des cas) et ont fait, ou ont le projet de refaire une formation par la suite. A contrario, lorsque la grossesse était un projet de couple (3/9), les femmes étaient déscolarisées depuis l'âge de 16 ans ou étaient dans une impasse scolaire. Aucune d'entre elles n'a repris les cours par la suite.

Concernant le devenir professionnel, on retrouve les mêmes catégories. En effet, les femmes ayant désiré leur grossesse s'avèrent avoir des emplois plus précaires, de courtes durées, voire n'ont jamais travaillé. Les autres femmes ont généralement pris une année pour s'occuper de leur enfant (4/6). Ensuite, elles ont toutes trouvé du travail, à plus ou moins long terme, mais leur période sans travail se révèle très courte. Certaines d'entre elles ont pris un congé maternité suite à leur deuxième grossesse qu'elles ont renouvelé au fil des autres grossesses.

\section{Ressenti sur leur parcours}

De manière globale, les femmes ont toutes bien vécu leur grossesse et ont une vision positive de leur parcours. Nous constatons également que pour certaines d'entre elles cette grossesse a eu un impact positif dans leur vie, leur permettant d'évoluer et de faire face aux difficultés qu'elles vivaient avant leur maternité (3/9).

De même aucune d'entre elles ne regrette avoir été mère adolescente. Mais il reste pour autant intéressant de noter que la moitié d'entre elles est dubitative voire catégorique 
sur le fait qu'elles ne réitéreraient pas l'expérience si elles avaient le choix (5/9). Il s'avère que $4 / 5$ des femmes concernées par cet avis, sont les femmes dont la grossesse n'était pas programmée et qui sont désormais séparées du père de l'enfant. Concernant les femmes qui le referaient sans hésiter, nous remarquons deux populations antagonistes : celles dont la grossesse était programmée et étaient déscolarisées à 16 ans $(2 / 4)$ et celles au contraire qui ont plutôt repris les cours et dont les grossesses n'étaient pas programmées (2/4).

Pour terminer, l'ensemble de ces mères ne souhaite pas que leurs enfants soient parents à l'adolescence. Certaines d'entre elles inciteraient même leur enfant à l'avortement ou du moins leur exposeraient les aspects négatifs de la parentalité précoce (3/9). De plus 7 femmes sur 9 ont un avis négatif sur les actuelles adolescentes enceintes.

\section{Le devenir obstétrical}

La majorité des femmes a eu trois ou quatre enfants (7/9). Nous notons que pour les deux femmes en ayant eu deux, les grossesses sont très espacées. Elles le justifient par le fait qu'elles ne se voyaient pas assumer un autre enfant rapidement après le premier.

À propos de la durée entre chaque grossesse, nous remarquons que la deuxième grossesse survient en moyenne trois ans plus tard. À chaque fois cette grossesse a été désirée et quand ça n'était pas le cas, les patientes ont eu recours à une IVG jugeant la charge d'une nouvelle parentalité trop lourde.

Pour les patientes ayant eu trois enfants ou plus, nous voyons des durées se démarquer. Le deuxième enfant est né en moyenne trois ans plus tard (4/9) ou alors 7 ans plus tard (2/9). Concernant la troisième grossesse, elle arrive principalement huit à dix ans après le premier enfant $(6 / 9)$.

\section{Devenir de l'enfant}

À propos de la relation parent-enfant, l'ensemble des mères nous dit avoir une bonne relation avec leur enfant. Ensuite, concernant la relation avec le père, lorsque le couple est séparé, l'enfant ne voit plus ou rarement son père $(3 / 4)$, a priori en raison du manque d'intérêt du géniteur pour son enfant.

Ensuite, concernant le développement de l'enfant, 6 enfants sur 9 ont une scolarité normale, 1 a redoublé sa classe préparatoire, 1 est en CLIS et 1 autre en Centre ressource de l'ouïe et de la parole (CROP) car il est malentendant. L'ensemble des mères souhaiterait que leurs enfants fassent de longues études, afin de pallier ce qu'elles n'ont pas pu réaliser.

Pour terminer, deux tiers des enfants sont en bonne santé «physique ». En effet, un enfant est atteint du syndrome de Turner, une autre est atteinte d'une Communication interauriculaire $(\mathrm{CIA})$ et un autre enfant est malentendant.

\section{Analyse et discussion}

\section{Limites et points forts de l'étude}

Tout d'abord, notre échantillon d'étude est restreint. En effet, l'étude n'a duré que 2 mois et demi, et le nombre de perdues de vue était très important. De plus, parmi les 20 personnes recontactées, 11 d'entre elles ont refusé l'entretien ou ne sont pas venues. On peut alors s' interroger sur les raisons de ce refus : sont-elles peu fières et peu confiantes à propos de leur parcours ? Si c'est le cas, notre étude n'est alors pas représentative de la multiplicité des anamnèses.

Malgré tout, avec un tel recul sur le devenir de ces femmes, cette étude reste une étude unique. Ce recul d'une décennie nous permet d'avoir un réel regard sur le devenir de ces femmes. Par ailleurs, si la population rencontrée n'est pas représentative de la diversité des cas, elle reste cependant hétérogène concernant les âges et la typologie des grossesses.

\section{Devenir des grossesses « insertion»}

Tout d'abord, concernant les relations familiales, on retrouve chez ces femmes des relations parentales difficiles. À propos des relations conjugales, on remarque que celles qui ne sont actuellement plus avec le père de l'enfant sont tout de même restées plusieurs années avec lui. Cette première union aura d'ailleurs abouti à la naissance de plusieurs enfants. Ces femmes forment des familles avec a minima 3 enfants.

Ensuite, ce sont des femmes qui sont dans une impasse scolaire ou une déscolarisation précoce. Aucune d'entre elles n'a de diplôme et elles ont des travails précaires voire une vie professionnelle inexistante.

Quant à leur vision sur leur parcours, la majorité n'a pas ressenti de difficulté dans leur nouveau rôle de mère et aucune n'a de regret sur cette parentalité précoce et elles recommenceraient sans hésiter.

\section{Les grossesses « identité »}

Concernant leur devenir social, les femmes de cette typologie ne sont plus en couple avec le père de l'enfant, et ce depuis la naissance. Cependant, les deux femmes concernées ont refait leur vie avec une personne depuis au moins 8 ans.

$\mathrm{Au}$ niveau scolaire, ces femmes ont arrêté les cours pendant leur grossesse sans reprendre leur scolarité immédiatement. Pour autant, chacune d'entre elles a suivi des formations ou obtenu des diplômes. De même, elles ont un parcours professionnel montrant peu de périodes de chômage.

$\grave{A}$ propos de leur ressenti, aucune ne regrette sa grossesse mais aucune de réitérerait l'expérience.

\section{Devenir des grossesses « accidentelles»}

La majorité des femmes est toujours en couple avec le même partenaire et a au moins 3 enfants aujourd'hui. Leurs relations familiales et amicales sont bonnes, et elles ont généralement gardé les mêmes relations.

À propos de leur devenir solaire, elles ont toutes fini leur année de cours, ou alors sont retournées à l'école par la suite ou ont reçu une formation a posteriori.

Par ailleurs, aucune ne regrette sa grossesse et la majorité recommencerait l'expérience si c'était à refaire. Enfin, toutes ont un ressenti positif sur leur parcours.

\section{Conclusion}

Ce travail nous a ainsi permis de nous rendre compte que le devenir des mères adolescentes n'est pas obligatoirement et systématiquement synonyme d'échec personnel et d'échec professionnel.

Pour les grossesses «insertion », ces femmes qui étaient dans une impasse scolaire il y a 10 ans n'ont repris ni les cours, ni de formation particulière et ont par conséquent 
un parcours professionnel précaire. Pour les grossesses « identités ", il ressort de nos entretiens que ces jeunes femmes se séparent presque systématique- ment du père de l'enfant. De même, il semble évident à ces femmes qu'elles ne réitéreraient pas l'expérience. Pour les grossesses «accidentelles », les jeunes femmes ne semblent pas se démarquer sur un point particulier, mais elles se rapprochent des deux autres typologies de grossesses sur certains points. Par exemple, tout comme les grossesses «identités », elles ont obtenu un diplôme.

Cependant, l'ensemble des femmes se regroupent sur un élément principal : la fierté à propos de leur parcours. Elles sont toutes fières de ce qu'elles ont réalisé et de ce qu'elles sont devenues. Une partie d'entre elles recommenceraient l'expérience. En revanche, il est paradoxal de voir qu'elles ont un avis négatif sur les jeunes filles actuellement.

\section{Remerciements}

Je remercie Mr Grandazzi Guillaume et Mme Brielle Nathalie pour m'avoir guidé tout le long de ce travail ainsi que l'ensemble des patientes qui a accepté de se confier à moi pour la réalisation de cette étude.

\section{Références}

[1] Définition de l'adolescence. Le Petit Larousse. [En ligne, consulté le 07/07/2017, disponible sur : http://www. larousse.fr/dictionnaires/francais/adolescence $/ 1156 ? q=$ adolescence\#1146].

[2] Santé de l'adolescent. OMS. [En ligne, consulté le 07/01/2016, disponible sur : http://www.who.int/ maternal_child_adolescent/topics/adolescence/fr/].

[3] Dadoorian D. Grossesses adolescentes. La vie de l'enfant. Toulouse: ERES; 2007 [136 pages].

[4] Le Van C. Les grossesses à l'adolescence ; Normes sociales, réalités vécues. L'Harmattan; 1998.

[5] CNGOF. Extrait des Mises à jour en Gynécologie Médicale. Grossesse et accouchement des adolescentes. [Consulté le 23/12/2014, en Ligne, disponible sur : http://www. cngof.asso.fr/d_livres/2003 gm 209_seince.pdf].

[6] Haesevoets Y-H. Traumatisme de l'enfance et de l'adolescence : un autre regard sur la souffrance psychique. Bruxelles: Édition De Boeck; 2008. p. 193-231.

[7] Berrewaerts J, Noirhomme-Renard F. Les grossesses adolescentes : quels sont les facteurs explicatifs identifiés dans la littérature ? Séries de dossiers techniques. Bruxelles: UCLRESO; 2006. p. 06-40.

[9] Le Van C. La grossesse à l'adolescence : un acte socialement déviant ? Adolescence 2006;55:225-34 [Consulté le 23/12/2014, en Ligne, disponible sur : http://www.cairn. info/revue-adolescence-2006-1-page-225.htm].

[10] Sellenet C, Portier-Le Cocq F. Maternités adolescentes : le temps bousculé. Rev Int Educ Famil 2013;3:17-36.

[11] Hoffman S. Teenage Chilbearing is not so bad after all... Or is it? A review of the new literature. Famil Plan Perspect 1998;30(5.).

[12] Bailey P, et al. Adolescent Pregnancy One year later: the effects of abortion vs. motherhood in north-east Brazil. J Adolescent Health 2001;29(3):223-32.

[13] Bettoli L. Parents mineurs : la grossesse, facteur de maturation pour les jeunes parents ? Quels risques comporte- t-elle ? Quel accompagnement à Genève ? Thérapie Familiale 2003;24:179-91. 